\title{
Recent Results on Quarkonium Spectroscopy and Exotic Quarkonium-like Resonances at Belle
}

\author{
Dan Santel ${ }^{* \dagger}$ \\ University of Cincinnati, the Belle Collaboration \\ E-mail: santeldmemail.uc.edu
}

In 2012, using a $121.4 \mathrm{fb}^{-} 1$ sample of data taken at the $\Upsilon(5 S)$ resonance, Belle reported the observation of two new charged bottomonium-like resonances $\left(Z_{b}^{ \pm}(10610)\right.$ and $\left.Z_{b}^{ \pm}(10650)\right)$ in the $M\left(\Upsilon(n S) \pi^{ \pm}\right)(\mathrm{n}=1,2,3)$ and $M\left(h_{b} \pi^{ \pm}\right) \quad(\mathrm{m}=1,2)$ distributions of $\Upsilon(5 S) \rightarrow \Upsilon(n S) \pi^{+} \pi^{-}$and $\Upsilon(5 S) \rightarrow h_{b}(m P)$ decays. We report the results of the search for neutral partners, " $Z_{b}^{0}$," in $\Upsilon(5 S) \rightarrow \Upsilon(n S) \pi^{0} \pi^{0}$, with evidence for the $Z_{b}^{0}(10610)$ at $4.9 \sigma$ with systematic errors. We also report the observation of the similar charged charmonium-like state $Z^{ \pm}(3895)$ in the $M\left(J / \psi \pi^{ \pm}\right)$ distribution in $Y(4260) \rightarrow J / \psi \pi^{+} \pi^{-}$decays.

XXI International Workshop on Deep-Inelastic Scattering and Related Subject -DIS2013, 22-26 April 2013

Marseilles, France

${ }^{*}$ Speaker.

On behalf of the Belle Collaboration 


\section{Bottomonium-like results at $\Upsilon(5 S)$}

The Belle Collaboration, using the Belle detector[8] at the KEKB asymmetric $e^{+} e^{-}$collider in Tsukuba, Japan has collected an integrated luminosity of $121.4 \mathrm{fb}^{-1}$ at $\sqrt{s} \approx 10.865 \mathrm{GeV}$, corresponding to the $b \bar{b}$ state $\Upsilon(5 S)$. Early analyses of $\Upsilon(5 S) \rightarrow \Upsilon(n S) \pi^{+} \pi^{-}(n=1,2,3)$ transitions measured a partial width two orders of magnitude greater than would be naively expected from the analogous measurements of $\Upsilon(m S) \rightarrow \Upsilon(n S) \pi^{+} \pi^{-}(m=2,3,4 ; n=1,2,3)$ [1]. Subsequently two new charged bottomonium-like resonances, $Z_{b}^{ \pm}(10610)$ and $Z_{b}^{ \pm}$(10650), were observed in decays of $\Upsilon(5 S) \rightarrow Z_{b} \pi \rightarrow \Upsilon(n S) \pi^{+} \pi^{-}\left(n=1,2,3\right.$ and $\Upsilon(5 S) \rightarrow Z_{b} \pi \rightarrow h_{b}(m P) \pi^{+} \pi^{-}(m=1,2)$. We report the results of a Dalitz plot analysis of $\Upsilon(5 S) \rightarrow \Upsilon(n S) \pi^{0} \pi^{0}(n=1,2,3)$ and the search for the neutral partners of the charged $Z_{b}$.

\section{Charmonium-like results at Belle}

The $Y(4260)$ resonance was observed initially by BaBar in initial state radiation (ISR) $e^{+} e^{-} \rightarrow$ $J / \psi \pi^{+} \pi^{-} \gamma_{I S R}$ decays [5], and was later confirmed by Belle [11]. Belle also reported evidence for a $Y$ (4008), but BaBar attributed the structure below the $Y(4260)$ to exponentially-decreasing nonresonant $J / \psi \pi^{+} \pi^{-}$. We report a new measurement of the $e^{+} e^{-} \rightarrow \gamma_{I S R} J / \psi \pi^{+} \pi^{-}$cross-section and a new measurement of $Y(4260)$ and $Y(4008)$ at Belle. We also report the observation of a new charged charmonium-like resonance $Z^{ \pm}(3895)$ in $Y(4260) \rightarrow Z \pi \rightarrow J / \psi \pi \pi$.

\section{The Belle detector}

The Belle detector consists of a silicon vertex detector (SVD), a 50-layer central drift chamber (CDC), an array of aerogel threshold Cherenkov counters (ACC), time-of-flight scintillation counters (TOF), and an electromagnetic calorimeter (ECL) comprising of $\mathrm{CsI}(\mathrm{Tl})$ crystals located inside a superconducting solenoid coil that provides a $1.5 \mathrm{~T}$ magnetic field. An iron flux-return located outside of the coil is used to detect $K_{L}$ mesons and to identify muons (KLM). The detector is described in detail elsewhere [8]. For charged hadron identification, a likelihood ratio is formed based on a dE/dx measurement in the CDC and the response of the ACC and TOF. Electron identification is based on a combination of $\mathrm{dE} / \mathrm{dx}$ measurement, the response of the ACC, and the position, shape, and total energy deposition of the shower detected in the ECL. Muons are identified by their range and transverse scattering in the KLM. We use a Geant-based Monte Carlo (MC) simulation [7] to model the response of the detector and determine the acceptance. The MC simulation includes run-dependent detector performance variations and background conditions.

\section{Selection criteria for $\Upsilon(5 S) \rightarrow \Upsilon(n S) \pi^{0} \pi^{0}$}

Selection criteria for the search for $Z_{b}^{0}$ are discussed in detail in Ref. [12]. The analysis uses a $121.4 \pm 1.9 \mathrm{fb}^{-1}$ data sample collected at the $\Upsilon(5 S)$ resonance. The $\Upsilon(5 S)$ candidates are reconstructed from $\Upsilon(n S) \pi^{0} \pi^{0} \quad(n=1,2)$, where the $\Upsilon(n S)$ candidates are reconstructed from $e^{+} e^{-}$and $\mu^{+} \mu^{-}$with invariant mass $M_{l^{+} l^{-}}$in the range $8-11 \mathrm{GeV}$. The additional channel $\Upsilon(2 S) \rightarrow \Upsilon(1 S)\left[l^{+} l^{-}\right] \pi^{+} \pi^{-}$is used for the $\Upsilon(2 S)$. Muon and electron candidates are required 
Table 1: Definition of the signal region and sideband region[12].

\begin{tabular}{lcc}
\hline \hline Final state & Signal region, GeV & Sideband region, Gev \\
\hline$\Upsilon(1 S)$ & $9.41<M_{\text {miss }}\left(\pi^{0} \pi^{0}\right)<9.53$ & $9.20<M_{\text {miss }}\left(\pi^{0} \pi^{0}\right)<9.35,9.60<M_{\text {miss }}\left(\pi^{0} \pi^{0}\right)<9.75$ \\
$\Upsilon(2 S)$ & $9.99<M_{\text {miss }}\left(\pi^{0} \pi^{0}\right)<10.07$ & $9.80<M_{\text {miss }}\left(\pi^{0} \pi^{0}\right)<9.95,10.15<M_{\text {miss }}\left(\pi^{0} \pi^{0}\right)<10.30$ \\
$\Upsilon(2 S) \rightarrow \Upsilon(1 S) \pi^{+} \pi^{-}$ & $10.00<M\left(\Upsilon \pi^{+} \pi^{-}\right)<10.05$ & $9.80<M\left(\Upsilon \pi^{+} \pi^{-}\right)<9.95,10.15<M\left(\Upsilon \pi^{+} \pi^{-}\right)<10.30$ \\
\hline \hline
\end{tabular}

to be positively identified, with standard impact parameter requirements. No particle identification (PID) requirement on the charged pions is used. The $\pi^{0}$ candidates are reconstructed from pairs of photons with $M_{\gamma \gamma}$ consistent with the nominal [16] $\pi^{0}$ mass. The combined quality of the $\pi^{0}$ mass-constrained fits $\left(\chi^{2}\left(\pi_{1}^{0}\right)+\chi^{2}\left(\pi_{2}^{0}\right)\right)$ is used to suppress the background.

The energy difference $\Delta E=E_{\text {cand }}-E_{C M}$ and momentum $P$ of the reconstructed $\Upsilon(5 S)$ in the center-of-mass (CM) frame are used to further suppress the background. The $e^{+} e^{-} \rightarrow l^{+} l^{-}(n) \gamma$ background is suppressed using the missing mass of the lepton-pair system, with $M_{\text {miss }}\left(l^{+} l^{-}\right)=$ $\sqrt{E_{C M}-E_{l^{+} l^{-}}^{2}-P_{l^{+} l^{-}}^{2}}$, where $E_{l^{+} l^{-}}$and $P_{l^{+} l^{-}}$are the energy and momentum of the $l^{+} l^{-}$system in the CM frame. In the event of multiple candidates (1-2\%), the candidate with the smallest $\chi^{2}\left(\pi_{1}^{0}\right)+\chi^{2}\left(\pi_{2}^{0}\right)$ is selected.

$\Upsilon(5 S) \rightarrow \Upsilon(n S)\left[l^{+} l^{-} \pi^{0} \pi^{0}\right.$ candidates are identified by the missing mass recoiling against the $\pi^{0} \pi^{0}$ system, $M_{\text {miss }}\left(\pi^{0} \pi^{0}\right)$. For $\Upsilon(5 S) \rightarrow \Upsilon(2 S)\left[\Upsilon(1 S) \pi^{+} \pi^{-}\right] \pi^{0} \pi^{0}$ decays, $\Upsilon(1 S)$ candidates are selected with $M_{l^{+} l^{-}}$within $150 \mathrm{MeV}$ of the nominal $\Upsilon(1 S)$ mass [16].

\section{Dalitz analysis of $\Upsilon(5 S) \rightarrow \Upsilon(n S) \pi^{0} \pi^{0}$}

To identify the $Z_{b}$ resonances, we perform a Dalitz plot analysis of the $\Upsilon(n S) \pi^{0} \pi^{0}$ events. Signal and sideband regions are defined in Table 1. The sideband regions are used to determine the background shape, which is parametrized as

$$
1+p_{1} \exp \left(-q_{1} s_{3}\right)+p_{2} \exp \left(-q_{2}\left(s_{\min }-a_{s}\right)\right)
$$

where $p_{1}, p_{2}, q_{1}, q_{2}$ are fit parameters and $s_{3}=M^{2}\left(\pi^{0} \pi^{0}\right), s_{\min }=\operatorname{Min}\left(s_{1}, s_{2}\right), s_{1,2}=M^{2}\left(\Upsilon(n S) \pi_{1,2}^{0}\right)$.

The amplitude analysis of $\Upsilon(5 S) \rightarrow \Upsilon(n S) \pi^{0} \pi^{0}$ is performed with an unbinned maximum likelihood function, where the amplitude is described by

$$
M\left(s_{1}, s_{2}\right)=A_{Z 1}\left(s_{1}, s_{2}\right)+A_{Z 2}\left(s_{1}, s_{2}\right)+A_{f 0}\left(s_{1}, s_{2}\right)+A_{f 2}\left(s_{1}, s_{2}\right)+A_{n r}\left(s_{1}, s_{2}\right),
$$

and $A_{Z 1}$ and $A_{Z 2}$ are amplitudes from the $Z_{b}^{0}(10610)$ and $Z_{b}^{0}(10650)$, respectively. The amplitudes $A_{f 0}, A_{f 2}$, and $A_{n r}$ describe the contributions in the $\pi^{0} \pi^{0}$ system from the $f_{0}(980), f_{2}(1270)$ and a non-resonant contribution, respectively. In cascades involving the $Z^{0}$, the pions are assumed to be emitted in an $S$-wave with respect to the heavy quarkonium system, preserving the orientation of its spin. The angular analysis in Ref [10] supports this assumption. The amplitudes of the $Z_{1}$ and $Z_{2}$ are thus parametrized with an $S$-wave Breit-Wigner

$$
B W(s, M, \Gamma)=\frac{\sqrt{M \Gamma}}{M^{2}-s-i M \Gamma} .
$$


Figure 1: One-dimensional projections of Dalitz plot fit results (open histograms) and experimental data (points with error bars) for $\Upsilon(1 S) \pi^{0} \pi^{0}$ (top) and $\Upsilon(2 S) \pi^{0} \pi^{0}$ (bottom) events in the signal region. Red and blue histograms show the fit with and without $Z_{b}^{0}$ resonances included. Shaded regions show the background from sidebands.[12]
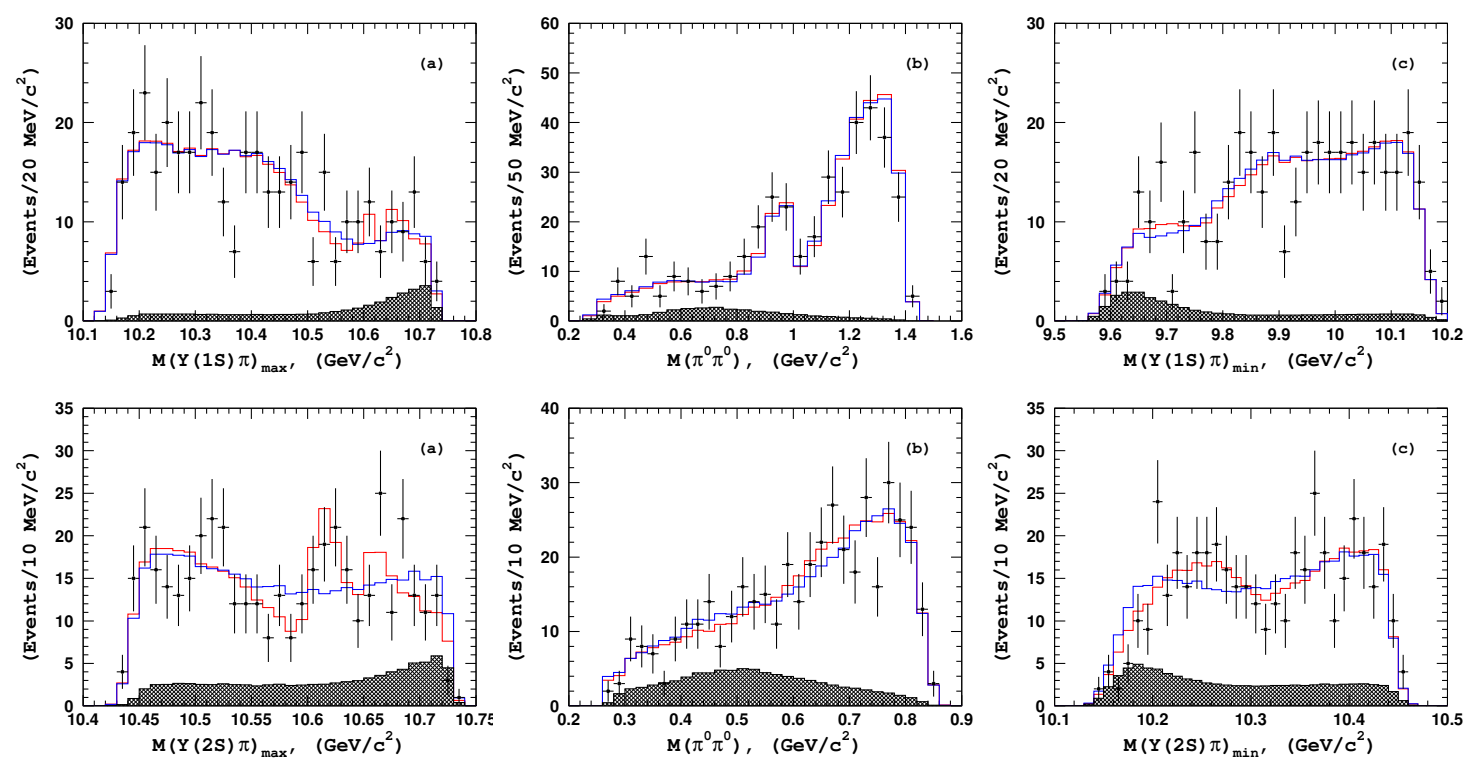

Masses and widths of the $Z_{b}^{0}$ are fixed to the results of the charged $Z_{b}$ analysis in Ref. [4]. The $f_{0}(980)$ is parameterized by a Flatte function[13], and the $f_{2}(1270)$ is parametrized by a BreitWigner. As suggested in Ref. [9], the non-resonant component $A_{n r}$ is parametrized by

$$
A_{n r}=A_{n r}^{1} e^{i \phi_{n r}^{1}}+A_{n r}^{2} e^{i \phi_{n r}^{2}} s_{3},
$$

where $A_{n r}^{1}=10$ and $\phi_{n r}^{1}=0$ are fixed, and the relative amplitudes and phases are free parameters in the fit.

The log likelihood function is defined as:

$$
\mathscr{L}=-2 \sum \log \left\{\varepsilon\left(s_{1}, s_{2}\right)\left(f_{\text {sig }} S\left(s_{1}, s_{2}\right)+\left(1-f_{\text {sig }} B\left(s_{1}, s_{2}\right)\right)\right)\right\},
$$

One-dimensional projections of the fit results are shown in Figure 1, which are similar to the corresponding $\Upsilon(n S) \pi^{+} \pi^{-}$results [4]. A $Z_{b}^{0}$ signal is most clearly seen in $M\left(\Upsilon(2 S) \pi^{0}\right)$ (Figure 1 , bottom left), with a statistical significance of $5.3 \sigma$ for the $Z_{b}^{0}(10610)$. Contributions from the $Z_{b}^{0}(10650)$ are not significant in either mode, but the data do not contradict its existence. Results of the fits are summarized in Table 2, and the multiple solutions to the fits are shown in Ref. [12].

The systematic uncertainties are dominated by the parametrization of the background PDF. The uncertainty of the background is determined by using different sideband sub-samples used to determine the PDF parameters and repeating the fit procedure. These sub-samples include: high- or low-mass sideband only, $\Upsilon(n S) \rightarrow e^{+} e^{-}$or $\Upsilon(n S) \rightarrow \mu^{+} \mu^{-}$events only. The statistical significance of the $Z_{b}^{0}(10610)$ in each fit is greater than $4.9 \sigma$. 
Table 2: Results of the Dalitz plot fit of $\Upsilon(5 S) \rightarrow \Upsilon(n S) \pi^{0} \pi^{0}$ events (Table from Ref [12]).

\begin{tabular}{lccccc}
\hline \hline Model & $\Upsilon(1 S) \pi^{0} \pi^{0}$ & $\Upsilon(1 S) \pi^{0} \pi^{0}$ & $\Upsilon(2 S) \pi^{0} \pi^{0}$ & $\Upsilon(2 S) \pi^{0} \pi^{0}$ & $\Upsilon(2 S) \pi^{0} \pi^{0}$ \\
\hline$A\left(Z_{1}^{0}\right)$ & with $Z_{b}^{0}$ & no $Z_{b}^{0}$ & with $Z_{b}^{0}$ & with $Z_{1}^{0}$ only & no $Z_{b}^{0}$ \\
$\phi\left(Z_{1}^{0}\right)$ & $0.50_{-0.30}^{+0.34}$ & 0.0 (fixed) & $0.58_{-0.14}^{+0.21}$ & $0.47_{-0.11}^{+0.15}$ & 0.0 (fixed) \\
$A\left(Z_{2}^{0}\right)$ & $-36 \pm 50$ & - & $-113 \pm 14$ & $-117 \pm 17$ & - \\
$\phi\left(Z_{2}^{0}\right)$ & $0.60_{-0.47}^{+0.51}$ & 0.0 (fixed) & $0.37_{-0.16}^{+0.20}$ & 0.0 (fixed) & 0.0 (fixed) \\
\hline$A\left(f_{2}\right)$ & $-59 \pm 60$ & - & $-125 \pm 27$ & - & - \\
$\phi\left(f_{2}\right)$ & $15.7 \pm 2.0$ & $14.6 \pm 1.6$ & $18.2 \pm 7.3$ & $23.9 \pm 7.3$ & $28.2 \pm 7.0$ \\
$A\left(f_{0}\right)$ & $60 \pm 11$ & $51 \pm 9$ & $36 \pm 21$ & $28 \pm 13$ & $28 \pm 10$ \\
$\phi\left(f_{0}\right)$ & $1.07 \pm 0.15$ & $0.97 \pm 0.12$ & $11.5 \pm 1.9$ & $10.5 \pm 1.9$ & $8.2 \pm 2.1$ \\
$A_{\mathrm{nr}}^{2}$ & $168 \pm 11$ & $163 \pm 10$ & $211 \pm 6$ & $213 \pm 7$ & $210 \pm 8$ \\
$\phi_{\mathrm{nr}}^{2}$ & $15.2 \pm 1.2$ & $13.9 \pm 0.7$ & $34.7 \pm 4.9$ & $31.8 \pm 4.3$ & $24.6 \pm 4.2$ \\
\hline$-2 \log \mathscr{L}$ & $162 \pm 4$ & $161 \pm 4$ & $80 \pm 12$ & $85 \pm 13$ & $93 \pm 15$ \\
\hline \hline
\end{tabular}

\section{Selection criteria for $e^{+} e^{-} \rightarrow J / \psi \pi^{+} \pi^{-} \gamma_{I S R}$}

The measurement of $\sigma\left(e^{+} e^{-} \rightarrow J / \psi \pi^{+} \pi^{-}\right)$between $3.8 \mathrm{GeV}$ and $5.5 \mathrm{GeV}$ is performed with the full Belle data sample with an integrated luminosity of $967 \mathrm{fb}^{-1}$, collected at or near the $\Upsilon(n S)$ $(n=1,2, \ldots, 5)$ resonances [14]. The $J / \psi$ candidates are reconstructed from $J / \psi \rightarrow e^{+} e^{-}$and $J / \psi \rightarrow \mu^{+} \mu^{-}$. We require four well-reconstructed charged tracks total with zero net charge.

Pion candidates are required to have a likelihood ratio $\mathscr{R}_{K}=\frac{\mathscr{L}_{K}}{\mathscr{L}_{K}+\mathscr{L}_{\pi}}<0.4\left(\mathscr{L}_{K / \pi}\right.$ is the likelihood of the kaon/pion hypothesis for a given candidate), with an identification efficiency of $95 \%$. Similar requirements are made for the likelihood ratios of the other tracks. For muon candidates, at least one must have $\mathscr{R}_{\mu}>0.95$. To suppress misidentified muon tracks when the second track has no muon identification, the polar angle of the two muons in the $\pi^{+} \pi^{-} \mu^{+} \mu^{-}$ CM frame must satisfy $\left|\cos \left(\theta_{\mu}\right)\right|<0.7$. For $J / \psi \rightarrow e^{+} e^{-}$events, at least one electron candidate must have $\mathscr{R}_{e}>0.95$ and the other $\mathscr{R}_{e}>0.05$. Events with photon conversions are removed by requiring $\mathscr{R}_{e}<0.75$ for the $\pi^{+} \pi^{-}$candidates. Selection criteria are described in detail in Ref. [11]. Candidate initial state radiation (ISR) events are identified by $\left|M_{\text {rec }}^{2}\right|<2.0 \mathrm{GeV}^{2}$, where $M_{\text {rec }}^{2}=\left(P_{C M}-P_{\pi^{+}}-P_{\pi^{+}-}-P_{l^{+}}-P_{l^{-}}\right)^{2}$, where $P_{i}$ is the four-momentum of the corresponding particle in the $e^{+} e^{-}$center of mass $(\mathrm{CM})$ frame. We define the $J / \psi$ signal region as $3.06 \mathrm{GeV}$ $<M\left(l^{+} l^{-}\right)<3.14 \mathrm{GeV}$, and the sidebands as $2.91 \mathrm{GeV}<M\left(l^{+} l^{-}\right)<3.03 \mathrm{GeV}$ and $3.17 \mathrm{GeV}$ $<M\left(l^{+} l^{-}\right)<3.29 \mathrm{GeV}$.

\section{The $J / \psi \pi^{+} \pi^{-}$cross-section}

The $\pi^{+} \pi^{-} l^{+} l^{-}$invariant mass distribution is shown in Figure 2 (left), including the estimated background using the normalized sidebands, and Figure 2 (right) shows the background-subtracted sample. Two enhancements are observed, identified as the $Y(4008)$ and $Y(4260)$, consistent with Ref. [11] but not with Ref. [5]. Systematic uncertainties of the cross section measurements are determined at $7.9 \%$ and $7.3 \%$ for $e^{+} e^{-}$and $\mu^{+} \mu^{-}$modes, respectively. The dominant uncertainties 
are from PID, measured from pure $\psi(2 S)$ events in the same data sample, at $4.7 \%$ and $3.6 \%$ for $e^{+} e^{-}$and $\mu^{+} \mu^{-}$modes, respectively. Tracking efficiency uncertainties are estimated to be $3.3 \%$ for both lepton modes. Systematic uncertainties are discussed in more detail in Ref. [14].
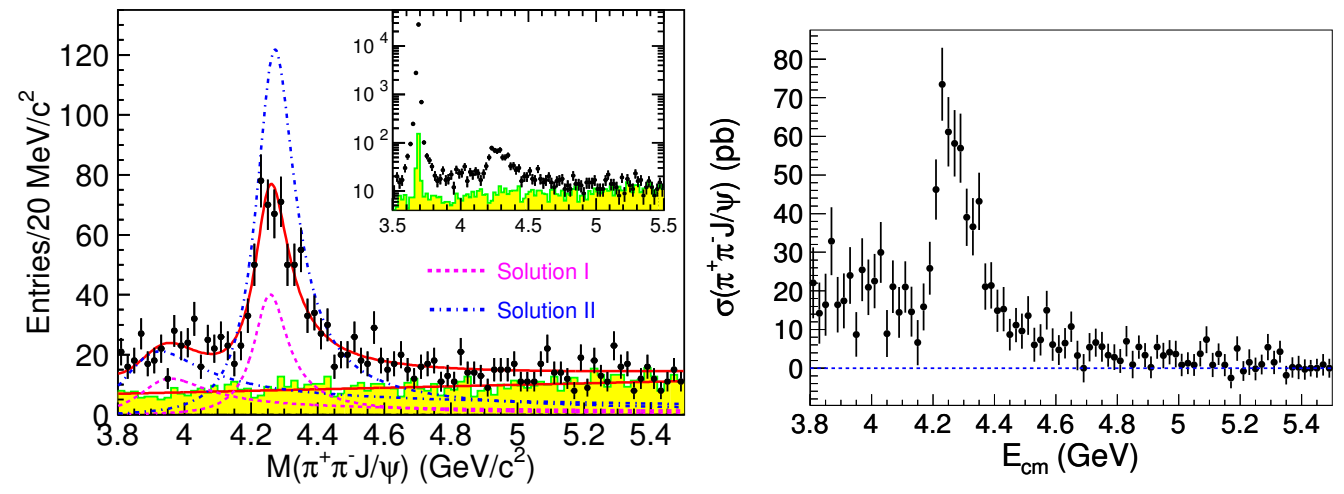

Figure 2: Left: Invariant mass distributions of $\pi^{+} \pi^{-} \ell^{+} \ell^{-}$. Points with error bars are data, and the shaded histograms are the normalized $J / \psi$ mass sidebands. The solid curves show the best fit with two coherent resonances background contribution. The dashed (dotted) curves are for solution I (II). The inset shows a logarithmic vertical scale, and the large peak around $3.686 \mathrm{GeV} / c^{2}$ is the $\psi(2 S) \rightarrow \pi^{+} \pi^{-} J / \psi$ signal. Right: Cross section of $e^{+} e^{-} \rightarrow \pi^{+} \pi^{-} J / \psi$ after background subtraction. The errors are statistical only.[14]

An unbinned maximum likelihood fit is performed to the $J / \psi \pi^{+} \pi^{-}$mass spectrum above 3.8 GeV. The PDF includes two coherent Breit-Wigner functions as in Ref. [11], for the two enhancements seen in the invariant mass spectrum and with the assumption of no continuum production of $e^{+} e^{-} \rightarrow J / \psi \pi^{+} \pi^{-}$. The background shape is fixed based on a linear fit to the sideband data. The fit results are shown in Figure 2.

There are two solutions of equal fit quality, corresponding to differing partial widths and relative phases of the two resonances. The masses and widths are the same in both solutions. Fit results are shown in Table 3. The fit quality of $\chi^{2} / n d f=101.6 / 84$ corresponds to a confidence level of $9.3 \%$. Systematic uncertainties in the resulting values for the fit parameters arise from the absolute energy scale, detection efficiency, background estimate, and parameterization of the resonances.

\section{Analysis of $Y(4260) \rightarrow J / \psi \pi^{+} \pi^{-}$}

The $Y(4260)$ signal region is defined by $4.15 \mathrm{GeV}<M\left(J / \psi \pi^{+} \pi^{-}\right)<4.45 \mathrm{GeV}$. Figure 3 shows projections of $M\left(\pi^{+} \pi^{-}\right)$and $M\left(J / \psi \pi^{ \pm}\right)$invariant mass distributions for the events in the $Y$ (4260) signal region, suggesting structures in both the $\pi^{+} \pi^{-}$and $J / \psi \pi$ systems. Background is again estimated from the normalized $J / \psi$ sidebands. The $M\left(\pi^{+} \pi^{-}\right)$distribution seems to match contributions from the $f_{0}(500), f_{0}(980)$, and $f_{2}(1270)$ resonances. Additional structure is evident in the $M(J / \psi)$ (identified hereafter as the $Z^{ \pm}(3895)$ ), interpreted as evidence for a charged charmonium-like state that decays to $J / \psi \pi^{ \pm}$

An unbinned maximum likelihood fit is performed to the $M_{\max }(J / \psi \pi)$ distribution, where $M_{\max }$ is the maximum of either $M\left(J / \psi \pi^{+}\right)$or $M\left(J / \psi \pi^{-}\right)$. The signal shape is parameterized as an $S$-wave Breit-Wigner function convolved with a Gaussian resolution function fixed at the MCestimated value $7.4 \mathrm{MeV}$, while the background is approximated by a cubic polynomial. The fit 
Table 3: Results of the fits to the $\pi^{+} \pi^{-} J / \psi$ mass spectrum with two coherent resonances. The parameter $\phi$ (in degrees) is the relative phase between the two resonances. The first and second uncertainties are statistical and systematic, respectively.[14]

\begin{tabular}{ccc}
\hline \hline Parameters & Solution I & Solution II \\
\hline$M\left(R_{1}\right)(\mathrm{MeV})$ & $3890.8 \pm 40.5 \pm 11.5$ \\
$\Gamma_{\text {tot }}\left(R_{1}\right)(\mathrm{MeV})$ & $254.5 \pm 39.5 \pm 13.6$ \\
$\Gamma_{e e} \mathscr{B}\left(R_{1} \rightarrow \pi^{+} \pi^{-} J / \psi\right)(\mathrm{MeV})$ & $(3.8 \pm 0.6 \pm 0.4)$ & $(8.4 \pm 1.2 \pm 1.1)$ \\
$M\left(R_{2}\right)(\mathrm{MeV})$ & \multicolumn{2}{c}{$4258.6 \pm 8.3 \pm 12.1$} \\
$\Gamma_{\text {tot }}\left(R_{2}\right)(\mathrm{MeV})$ & $134.1 \pm 16.4 \pm 5.5$ \\
$\Gamma_{e e} \mathscr{B}\left(R_{2} \rightarrow \pi^{+} \pi^{-} J / \psi\right)(\mathrm{MeV})$ & $(6.4 \pm 0.8 \pm 0.6)$ & $(20.5 \pm 1.4 \pm 2.0)$ \\
$\phi(\mathrm{rad})$ & $59 \pm 17 \pm 11$ & $-116 \pm 6 \pm 11$ \\
\hline \hline
\end{tabular}
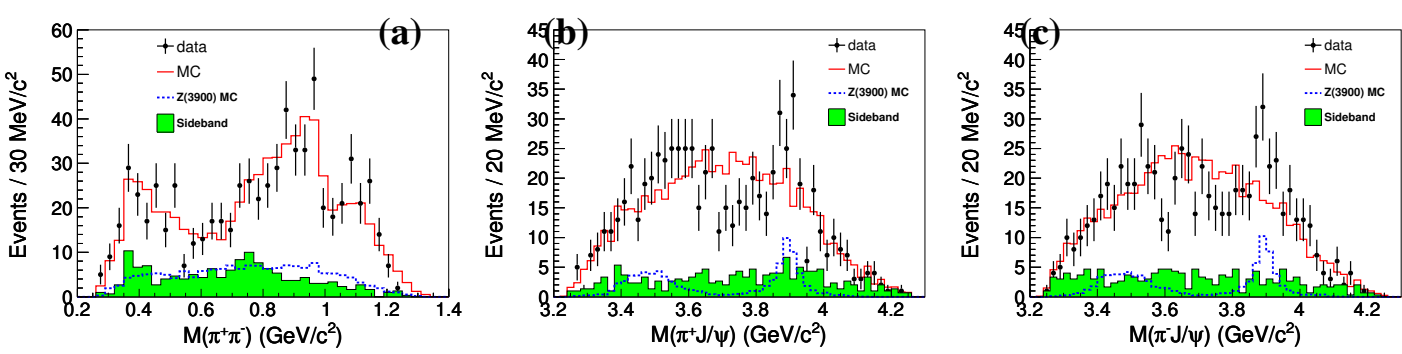

Figure 3: Invariant mass distributions of (a) $\pi^{+} \pi^{-}$, (b) $\pi^{+} J / \psi$ and (c) $\pi^{-} J / \psi$ for events in the $Y$ (4260) signal region, for signal and sideband data (points and filled histograms) and Monte Carlo (closed histograms).[14]

yields $159 \pm 49 \pm 7 Z^{ \pm}(3895)$ events, with a mass $3894.5 \pm 6.6 \pm 4.5 \mathrm{MeV}$ and a width $63 \pm 24 \pm 26$ $\mathrm{MeV}$, where the errors are statistical and systematic, respectively. The largest contributions to the systematic uncertainties arise from the parameterizations of signal and background shapes, mass resolution, and mass calibration. The statistical significance of the $Z^{ \pm}(3895)$ is determined to be $5.5 \sigma$, and greater than $5.2 \sigma$ in all alternate fits from systematics checks.

\section{Summary}

In a Dalitz plot analysis of $\Upsilon(5 S) \rightarrow \Upsilon(n S) \pi^{0} \pi^{0}(\mathrm{n}=1,2)$, a $Z_{b}^{0}(10610)$ signal is most clearly seen in $M\left(\Upsilon(2 S) \pi^{0}\right)$, with a statistical significance of $5.3 \sigma$. Contributions from the $Z_{b}^{0}(10650)$ are not significant in either mode, but the data do not contradict its existence either. Available statistics are not significant for observation of this state. With systematic uncertainties, the significance of the $Z_{b}^{0}(10610)$ signal is greater than $4.9 \sigma$.

In the analysis of $\sigma\left(e^{+} e^{-} \rightarrow \gamma_{I S R} J / \psi \pi^{+} \pi^{-}\right)$, new measurements of the previously seen states $Y(4008)$ and $Y(4260)$ are obtained, and an observation of the new charged charmonium-like state $Z^{ \pm}(3895)$ is reported. As these results were being prepared for publication, the BESIII collaboration also reported an observation of the $Z^{ \pm}(3895)$ [15]. 


\section{References}

[1] K.-F. Chen, W.-S. Hou, M. Shapkin, A. Sokolov et al. (The Belle collaboration), PRL 100, 112001 (2008)

[2] K.-F. Chen, W.-S. Hou et al. (The Belle collaboration), PRD 82, 091106(R) (2010).

[3] R. H. Dalitz, Philosophical Magazine 44: 1068. doi:10.1080/14786441008520365 (1953).

[4] A. Bondar, A. Garmash, R. Mizuk, D. Santel, K. Kinoshita, et al. (Belle collaboration), PRL 108, 122001 (2012).

[5] B. Aubert et al. (BABAR Collaboration), PRL 95, 142001 (2005).

[6] S.-K. Choi, S.L. Olsen et al. (The Belle Collaboration), PRL 100, 142001 (2008).

[7] R. Brun et al., GEANT 3.21, CERN DD/EE/84-1, 1984.

[8] A. Abashian et al. (The Belle Collaboration), Nucl. Instrum. Methods Phys. Res., Sect. A 479, 117 (2002).

[9] M.B. Voloshin, Prog. Part. Nucl. Phys. 61, 455 (2008). M. B. Voloshin, Phys. Rev. D 74, 054022 (2006) and references therein.

[10] I. Adachi et al. (The Belle Collaboration), arXiv:1105.4583.

[11] C. Z. Yuan et al. (The Belle Collaboration), PRL 99, 182004 (2007).

[12] P. Krokovny et al. (The Belle Collaboration), arXiv:1207.4345.

[13] S.M. Flatté Phys. Lett. B 63, 224, (1976).

[14] Z. Q. Liu, C.P. Shen C. Z. Yuan et al. (The Belle Collaboration), PRL 110, 252002 (2013)

[15] The BESIII Collaboration, arXiv:1303.5949

[16] J. Beringer et al. (Particle Data Group), PRD 86, 010001 (2012). 